Laboratoire d'Arithmétique, Calcul formel et d'Optimisation

UMR CNRS 6090

\title{
Lower Semicontinuous Regularization for Vector-Valued Mappings
}

\section{Aït Mansour \\ A. Metrane \\ M. Théra}

Rapport de recherche $\mathrm{n}^{\circ}$ 2004-06

Déposé le 1 juin 2004 
Université de Limoges, 123 avenue Albert Thomas, 87060 Limoges Cedex

Tél. (33) 555457323 - Fax. (33) 555457322 - laco@unilim.fr http://www.unilim.fr/laco/ 


\title{
LOWER SEMICONTINUOUS REGULARIZATION FOR VECTOR-VALUED MAPPINGS
}

\author{
M. AIT MANSOUR, A. METRANE AND M. THÉRA \\ Dedicated to Alex Rubinov
}

\begin{abstract}
The concept of the lower limit for vector-valued mappings is the main focus of this work. We first introduce a new definition of adequate lower and upper level sets for vector-valued mappings and establish some of their topological and geometrical properties. Characterization of semicontinuity for vector-valued mappings is thereafter presented. Then, we define the concept of vector lower limit, proving its lower semicontinuity, and furnishing in this way a concept of lower semicontinuous regularization for mappings taking their values in a complete lattice. The results obtained in the present work subsume the standard ones when the target space is finite dimensional. In particular, we recapture the scalar case with a new flexible proof. In addition, extensions of usual operations of lower and upper limits for vector-valued mappings are explored. The main result is finally applied to obtain a continuous D.C. decomposition of continuous D.C. mappings.
\end{abstract}

\section{INTRODUCTION}

The concept of lower semicontinuity introduced for scalar functions by R. Baire is a fundamental ingredient in different fields of mathematical analysis. It has been used in different contexts and in particular by D. Hilbert and L. Tonelli in the calculus of variations. The increasing

2000 Mathematics Subject Classification. Primary 47A15; Secondary 46A32, 47D20.

Key words and phrases. Vector-valued mappings, vector lower limit, lower semicontinuous regularization, lower level set, D.C.-mappings.

The research of Mohamed Ait Mansour was supported by AUF, Conseil Régional du Limousin and by Laboratoire d'Arithmétique, Calcul Formel et Optimisation, Université de Limoges.

The first author is grateful to Professor Nicolae Popovici for his useful remarks and discussions on the first version of the present work. 
developments of optimization theory, especially Pareto optimization has manifested the strong need to extend this concept to vector-valued mappings. This has motivated a quite number of mathematicians to tackle this topic. The first efforts in this direction go back to Théra [21] (1978), G. Gierz et al [9] (1980), Penot and Théra [17] (1982), G. Gerrits [8] (1985), H. Holwerda [10] (1989), D.T. Luc [12] (1989), J. M. Borwein and Théra [3] (1990), Combari, Laghdir and Thibault [4] (1994). We refer also to the recent contributions of M. Akian [1] (1999) and M. Akian and I. Singer [2] (2003).

A basic fact in real analysis is that every real-valued function $f$ not necessarily lower semi-continuous, admits a lower semicontinuous regularization, l.s.c regularization for short, defined by means of the lower limit of $f$ :

$$
\bar{f}(x):=\liminf _{y \rightarrow x} f(y) .
$$

A very natural and challenging question is, therefore, to determine a concept of l.s.c regularization for vector-valued mappings. It seems that, since the contribution of Théra [21], in which he provided some types of l.s.c regularizations for mappings with values in order complete Daniell spaces and lattices Daniell spaces, a little bit of attention has been focused on the topic. Therefore, there is still a need to advance this direction in the general setting of mappings with values in partially ordered spaces not necessarily Daniell.

The main scope of this paper is then to define an appropriate l.s.c regularization for mappings with values in a complete Banach lattice. Thus, after defining a suitable lower limit, we will mostly be devoted in proving its semicontinuity.

On the way to do it, inspired by the ideas of Penot and Théra [17] and motivated by Combari et al [4], we introduce a concept of lower and upper "level" sets. We first study these sets, show that they own nice properties, both topological and geometrical, and establish the link between them and semicontinuity. Then, we succeed in defining the concept of lower limit for a vector-valued mapping $f$ at a point $x$ in its domain. We will use the notation $v$ - $\liminf _{y \rightarrow x} f(y)$ rather than the standard one $\liminf _{y \rightarrow x} f(y)$ in order to do make clear that we are in the framework of vector-valued mappings. 
We now outline the plan of the remaining contents of the work, which we organize in nine sections. Section 2 includes the notations and most necessary definitions used later. Section 3 introduces adequate lower and upper level sets and illustrates them by some examples. The fourth section deals with characterizations of semicontinuity for vector-valued mappings. Section 5 presents a study of some of the topological and geometrical properties of the lower level set. In the following Section, 6 , we reach our goal by proving that the vector lower limit we consider defines a l.s.c regularization for a given vector-valued map. We begin with Hilbert-valued maps and then consider Banach lattice-valued ones. In Section 7 we check that our contribution can be viewed as an extension of standard results. In particular, we recapture the scalar case with a more flexible proof. In Section 8, we extend the usual operations of estimation of lower and upper limits of the sum of two vector-valued mappings. Section 9 aims to apply the main result to obtain a continuous decomposition for D.C.-mappings in the vector case, which extends some previous results in the literature.

\section{NotATIONS AND DEFINITIONS}

Throughout this paper, $E$ and $F$ are real-vector topological spaces. For a subset $S$ in $E$ or $F$, Int $S$ and cl $S$ denote the interior and the closure of $S$, respectively. Let $C \subset F$ be a closed and convex cone, which is supposed to be pointed, that is $C \cap-C=\{0\}$, and with nonempty interior. The cone $C$ defines a partial order on $F$ denoted by $\leq_{c}$ and defined by

$$
y_{1} \leq_{c} y_{2} \Leftrightarrow y_{2} \in y_{1}+C .
$$

The positive polar cone $C_{+}^{*}$ of $F$ is defined by

$$
C_{+}^{*}=\left\{y^{*} \in F^{*}:\left\langle y^{*}, y\right\rangle \geq 0 \text { for all } y \in C\right\},
$$

where $F^{*}$ is the continuous dual of $F$ and $\langle.,$.$\rangle the corresponding duality$ pairing. The set $C_{0}$ used in the sequel is defined by

$$
C_{0}:=\left\{w \in C: \xi(w)>0, \forall \xi \in C_{+}^{*} \backslash\{0\}\right\} .
$$

Remark 2.1. Notice that we have Int $C \subset C_{0}$, we refer for instance to [15].

$F^{\bullet}$ will stand for $F \cup\{+\infty\}$, where $+\infty$ denotes the greatest element of $F$ with respect to the order $\leq_{c}$. We will write $x<_{c} y$, for $x, y \in E$, if $y-x \in \operatorname{Int} C$. The order between subsets in $F$ is defined as follows:

Definition 2.2. Let $A$ and $B$ be two subsets of $F^{\bullet}$. We use the notation $A \leq_{c} B$, if for each $x \in A$ and each $y \in B$, we have $y-x \in C$. 
It is equally worth to recall that a subset $A$ of $F$ is said to be directed upwards if for every $a, b$ in $A$ there exists $c \in A$ such that $a \leq_{c} c$ and $b \leq_{c} c$. By analogy, directed downwards subsets can be defined.

For a given subset $A \subset F$, there may, or may not, be $a \in F$ with the following property :

for every $c \in F, a \leq_{c} c$ if and only if $b \leq_{c} c$ for every $b \in A$,

that is, $a \leq_{c} c$ if, and only if, $c$ is an upper bound for $A$. Obviously, if $a$ exists it is unique; it is called the least upper bound of $A$ and denoted by $\sup _{\leq_{c}} A$ or simply sup $A$ if there is no risk of confusion on the order. In a similar way, $\inf A$, whenever it exists, is called the greatest lower bound of $A$ and it is the element of $F$ such that

for every $c \in F, c \leq_{c} \inf A$ if and only if $c \leq_{c} b$ for every $b \in A$.

We shall recall that $F$ is called a lattice whenever $\sup \{a, b\}$ and $\inf \{a, b\}$ exist for all elements $a, b$ in $F$. It follows that finite subsets in a lattice have a least upper bound and a greatest lower bound. Finally, a lattice such that non-empty subsets bounded from above and directed upwards have a least bound are called complete lattice or else Dedekind lattice ${ }^{1}$. We point out here that no confusion should be done between complete lattice and totally ordered spaces.

The domain of a function, $f: E \rightarrow F^{\bullet}$, is denoted by $\operatorname{Dom} f$ and is defined by

$$
\operatorname{Dom} f=\left\{x \in E \mid f(x)<_{c}+\infty\right\},
$$

and its epigraph by

$$
\text { epi } f=\{(x, y) \in E \times F \mid y \in f(x)+C\} .
$$

Recall now the following definitions:

Definition 2.3. $f$ is said to be $C$-convex, if for every $\alpha \in[0,1]$ and $x_{1}, x_{2} \in E$ one has

$$
\alpha f\left(x_{1}\right)+(1-\alpha) f\left(x_{2}\right) \in f\left(\alpha x_{1}+(1-\alpha) x_{2}\right)+C .
$$

Definition 2.4. A mapping $f: E \rightarrow F$ is said to be $C$-D.C., if there exist two $C$-convex mappings $g$ and $h$ such that:

$$
f(x)=g(x)-h(x) \quad \forall x \in E .
$$

The pair $(g, h)$ of $C$-convex maps will be called a $C$-D.C. decomposition of $f$.

We recall now the definitions of lower semicontinuity and sequential lower semicontinuity of a vector-valued mapping introduced respectively in [17] and [4].

\footnotetext{
${ }^{1}$ Some authors use the terminology of conditionally complete lattice as in [2].
} 
Definition 2.5. [17] A mapping $f: E \rightarrow F^{\bullet}$ is said to be lower semicontinuous (l.s.c) at $\bar{x} \in E$, if for any neighborhood $V$ of zero and for any $b \in F$ satisfying $b \leq_{c} f(\bar{x})$, there exists a neighborhood $U$ of $\bar{x}$ in $E$ such that

$$
f(U) \subset b+V+C \cup\{+\infty\} .
$$

Remark 2.6. Following [17], if $f(\bar{x}) \in F$ then Definition 2.5 amounts to saying that for all neighborhood $V$ of zero (in $F$ ), there exists a neighborhood $U$ of $\bar{x}$ such that

$$
f(U) \subset f(\bar{x})+V+C \cup\{+\infty\} .
$$

Definition 2.7. [4] A mapping $f: E \rightarrow F$ is said to be sequentially lower semicontinuous (s-l.s.c) at $\bar{x} \in E$, if for any $b \in F$ satisfying $b \leq_{c} f(\bar{x})$ and for any sequence $\left(x_{n}\right)$ of $E$ which converges to $\bar{x}$, there exists a sequence $\left(b_{n}\right)$ (in $F$ ) converging to $b$ and satisfying $b_{n} \leq_{c} f\left(x_{n}\right)$, for every $n \in \mathbb{N}$.

Remark 2.8. For $\bar{x} \in$ Dom $f$, Definition 2.7 can be expressed simply as follows: For each sequence $\left(x_{n}\right)$ converging to $\bar{x}$, there exists a sequence $\left(b_{n}\right)$ converging to $f(\bar{x})$ such that $b_{n} \leq_{c} f\left(x_{n}\right)$ for all $n \in \mathbb{N}$.

Note that it has been proved in [4] that Definitions 2.5 et 2.7 coincide whenever $E$ and $F$ are metrizable.

Definition 2.9. $F$ is said to be normal if $F$ has a basis of order-convex neighborhood of zero of the form $V=(V+C) \cap(V-C)$.

Remark 2.10. It is worth mentioning as well that:

- The sequential upper semicontinuity of $f$ (s-u.s.c for brevity) is defined by saying that $-f$ is s-l.s.c.

- If $(F, C)$ is normal, one may check that $f$ is sequentially continuous at $\bar{x} \in E$ with $f(\bar{x}) \in F$, if and only if $f$ is s-l.s.c and s-u.s.c at $\bar{x}$.

- Whenever $E$ is metrizable and $F=\mathbb{R}$, the s-l.s.c continuity coincides with the classical lower semicontinuity. In this case, a function is s-l.s.c at every point of $E$ if, and only if its epigraph is closed in $E \times F$.

- Note that every l.s.c vector-valued mapping has a closed epigraph (see [3]), but the converse is not true as the following counterexample furnished in [17] shows:

The mapping $h: \mathbb{R} \rightarrow \mathbb{R}^{2}$ defined by :

$$
h(x)=\left\{\begin{array}{cl}
(0,0) & \text { if } x=0 \\
\left(\frac{1}{|x|},-1\right) & \text { otherwise }
\end{array}\right.
$$


is not not l.s.c at $(0,0)$ while its epigraph (with respect to $C=$ $\left.\mathbb{R}_{+}^{2}\right)$ is closed.

We end up these preliminaries by recalling that the lower part of Painlevé-Kuratowski set-convergence, of a sequence $\left(A_{n}\right)$ of subsets of $F$, is given by

$\liminf _{n} A_{n}=\left\{y \in F: y=\lim _{n} y_{n}\right.$, there exists $n_{0}:$ for all $\left.n \geq n_{0}, y_{n} \in A_{n}\right\}$.

The sequence $\left(A_{n}\right)$ will be said lower convergent to $A \subset F$ in the sense of Painlevé-Kuratowski if, and only if

$$
A \subset \liminf _{n} A_{n}
$$

\section{Adequate Local LOWer AND Upper LeVEl Sets}

In the present section, on the way to our objective, we introduce adequate notions of local lower and upper "level" sets for vector-valued mappings defined from $E$ into $F^{\bullet}$.

Definition 3.1. Let $f$ be an extended-vector-valued mapping and $\bar{x} \in \operatorname{Dom} f$. Denoting by $\vartheta(\bar{x})$ the family of neighborhoods of $\bar{x}$, we introduce the following "level" sets:

$$
A_{\bar{x}}^{f}:=\{y \in F \mid \forall V \in \vartheta(y), \exists U \in \vartheta(\bar{x}), f(U) \subset V+C \cup\{+\infty\}\} ;
$$

$$
B_{\bar{x}}^{f}:=\{y \in F \mid \forall V \in \vartheta(y), \exists U \in \vartheta(\bar{x}), f(U) \subset V-C \cup\{+\infty\}\} ;
$$

$$
s-A_{\bar{x}}^{f}:=\left\{y \in F \mid \forall\left(x_{n}\right) \rightarrow \bar{x}, \exists\left(b_{n}\right) \rightarrow y, \quad b_{n} \leq_{c} f\left(x_{n}\right) \quad \forall n \in \mathbb{N}\right\} ;
$$

$$
s-B_{\bar{x}}^{f}:=\left\{y \in F \mid \forall\left(x_{n}\right) \rightarrow \bar{x}, \exists\left(b_{n}\right) \rightarrow y, \quad b_{n} \geq_{c} f\left(x_{n}\right) \quad \forall n \in \mathbb{N}\right\} .
$$

Remark 3.2. In order to illustrate these definitions we present the following examples:

\section{Example 1:}

Let $f$ be the real-valued function defined by:

$$
f(x)= \begin{cases}x & \text { if } x<0 \\ x+1 & \text { otherwise }\end{cases}
$$

Note that $f$ is not lower semicontinuous and observe that

$$
A_{x}^{f}= \begin{cases}]-\infty, x] & \text { if } x \leq 0 \\ ]-\infty, x+1] & \text { otherwise }\end{cases}
$$


Remark 3.3. Let us point out that for any extended real-valued function $\left.f, \operatorname{cl} A_{\bar{x}}^{f}=\right]-\infty, \liminf _{x \rightarrow \bar{x}} f(x)$ ]. In Section 7 we present the proof in details for finite dimensional-valued functions.

\section{Example 2:}

Let $H$ be a separable Hilbert space and let $\left(e_{n}\right)_{n \in \mathbb{N}}$ be a basis of $H$. We suppose that the order is defined by the closed convex cone $H_{+}$given by

$$
H_{+}=\left\{x \in H \mid\left\langle e_{p}, x\right\rangle \geq 0, \forall p \in \mathbb{N}\right\}
$$

Denoting $\left\langle e_{i}, f\right\rangle$ by $f_{i}$, we consider the function $f: \mathbb{R} \longrightarrow H$ defined by

$$
\left\{\begin{array}{l}
f_{1}(x)=\left\{\begin{array}{ll}
0 & \text { if } x<0 \\
1 & \text { otherwise }
\end{array} \quad \text { if } i \neq 1 .\right.
\end{array}\right.
$$

We observe that $f$ is lower semicontinuous everywhere except at 0 , and we check that ${ }^{2}$

$$
A_{x}^{f}=\prod_{p=1}^{\infty}\left\langle e_{p}, A_{x}^{f}\right\rangle
$$

Indeed,

$$
\left\langle e_{p}, A_{x}^{f}\right\rangle=A_{x}^{\left\langle e_{p}, f\right\rangle}
$$

Then, for $p>1$, we have

$$
\left.\left.A_{x}^{\left\langle e_{p}, f\right\rangle}=A_{x}^{f_{p}}=\right]-\infty, 0\right] .
$$

For $p=1$, we see that

$$
\left.\left.A_{x}^{\left\langle e_{1}, f\right\rangle}=A_{x}^{f_{1}}=\right]-\infty, 0\right] .
$$

Hence,

$$
\left.\left.A_{x}^{f}=\prod_{p=1}^{\infty}\left\langle e_{p}, A_{x}^{f}\right\rangle=\prod_{p=1}^{\infty}\right]-\infty, 0\right]=-H_{+} .
$$

\section{Example 3:}

Consider the mapping $f=\left(f_{1}, f_{2}\right): \mathbb{R} \longrightarrow \mathbb{R}^{2}$ defined by

$$
\left\{\begin{array}{l}
f_{1}(x)=\left\{\begin{array}{cc}
0 & \text { if } x<0 \\
1 & \text { otherwise. }
\end{array}\right. \\
f_{2}(x)=x
\end{array}\right.
$$

A straightforward calculation shows that $\left.\left.\left.\left.\operatorname{cl} A_{x}^{f}=\right]-\infty, 0\right] \times\right]-\infty, x\right]$ if $x \leq 0$ and $\left.\left.\left.\left.\operatorname{cl} A_{x}^{f}=\right]-\infty, 1\right] \times\right]-\infty, x\right]$ otherwise.

\footnotetext{
$2_{\text {as }}$ it is done later in Lemma 6.2.
} 
Remark that $\operatorname{cl} A_{x}^{f}$ is convex, directed upwards and in addition we have

$$
A_{x}^{f}=A_{x}^{f}-\mathbb{R}_{+}^{2} .
$$

In Section 5, we will prove that these properties of this lower "level" set hold in general.

In the next section, we characterize the semicontinuity of vectorvalued mappings in terms of the above level sets.

\section{Characterization of Semicontinuity For VECTOR-MAPPINGS}

We begin with the following proposition which gives the link between the level sets and the s-l.s.c, l.s.c, s-u.s.c and u.s.c.

Proposition 4.1. Let $f$ be an extended vector map and $\bar{x} \in \operatorname{Dom} f$. Then,

(1) $f$ is s-l.s.c at $\bar{x}$ if, and only if, $f(\bar{x}) \in s-A_{\bar{x}}^{f}$;

(2) $f$ is l.s.c en $\bar{x}$ if, and only if, $f(\bar{x}) \in A_{\bar{x}}^{f}$;

(3) $f$ is s-u.s.c at $\bar{x}$ if, and only if, $f(\bar{x}) \in s-B_{\bar{x}}^{f}$;

(4) $f$ is s.c.s at $\bar{x}$ if, and only if, $f(\bar{x}) \in B_{\bar{x}}^{f}$.

Proof. The proof follows from the definitions.

Now, we can determine the level sets of semicontinuous extendedvector-valued mappings. First, we establish the equivalence between s- $A_{\bar{x}}^{f}$ and $A_{\bar{x}}^{f}$ once $E$ and $F$ are metrizable.

Proposition 4.2. Assume that $E$ and $F$ are metrizable. Let $f: E \rightarrow$ $F^{\bullet}, \bar{x} \in \operatorname{Dom} f$. Then, we have

$$
s-A_{\bar{x}}^{f}=A_{\bar{x}}^{f} \quad \text { and } \quad s-B_{\bar{x}}^{f}=B_{\bar{x}}^{f} .
$$

Proof. Let $y \in s-A_{\bar{x}}^{f}$ and consider $y \notin A_{\bar{x}}^{f}$. There exists a neighborhood $V$ of zero (in $F$ ) such that for every neighborhood $U$ of $\bar{x}, f(U)$ is not included in $y+V+C \cup\{+\infty\}$. For $n \in \mathbb{N}^{*}$, take $U_{n}=B\left(\bar{x}, \frac{1}{n}\right)$ and select $x_{n} \in B\left(\bar{x}, \frac{1}{n}\right)$ such that

$$
f\left(x_{n}\right)-y \notin V+C \cup\{+\infty\} .
$$

As $\left(x_{n}\right)$ converges to $\bar{x}$ and $y \in s-A_{\bar{x}}^{f}$, there exists a sequence $\left(b_{n}\right)$ in $F$ such that

$$
\lim _{n \rightarrow+\infty} b_{n}=y \quad \text { and } \quad b_{n} \leq_{c} f\left(x_{n}\right), \quad \forall n \in \mathbb{N} .
$$


Thus, for $n$ large enough i.e, $n \geq N$, we get

$$
b_{n}-y \in V, \quad \forall n \geq N .
$$

Using (4.3) and the fact that

$$
b_{n}-y \leq_{c} f\left(x_{n}\right)-y, \quad \forall n \geq N,
$$

we obtain

$$
f\left(x_{n}\right)-y \in V+C \cup\{+\infty\}, \quad \forall n \geq N,
$$

a contradiction with (4.1). Then $y \in A_{\bar{x}}^{f}$.

Conversely, let $y \in A_{\bar{x}}^{f}$. Then, for each neighborhood $V$ of origin, there exists a neighborhood $U$ of $\bar{x}$ such that

$$
f(U) \subset y+V+C \cup\{+\infty\} .
$$

For each integer $k \geq 1$, take $V_{k}=B\left(0, \frac{1}{k}\right)$ and select a neighborhood $U_{k}$ of $\bar{x}$ verifying

$$
f\left(U_{k}\right) \subset y+B\left(0, \frac{1}{k}\right)+C \cup\{+\infty\} .
$$

Let $\left(x_{n}\right)$ be a sequence converging to $\bar{x}$. For every $k \geq 1$, there exists an integer $N_{k}$ such that

$$
\forall n \geq N_{k}, \quad x_{n} \in U_{k} .
$$

Consider the following sequence of integers $S(k)$ :

$$
\begin{aligned}
S(1) & =N_{1} \\
S(k+1) & =\max \left[S(k)+1, N_{k+1}\right] .
\end{aligned}
$$

(Remark that $S(k)$ is strictly increasing). For some $k \in \mathbb{N}^{*}$, for $n \in$ $\{S(k), \ldots, S(k+1)-1\}$, taking into account relations (4.4) and (4.5), we can pick an element $v_{n, k} \in B\left(0, \frac{1}{k}\right)$ such that

$$
y+v_{n, k} \leq_{c} f\left(x_{n}\right) .
$$

Now, for each integer $n \neq 0$ such that $n<S(1)$, pick an element $b_{n} \in F$ such that $b_{n} \leq_{c} f\left(x_{n}\right)$. The sequence $\left(y_{n}\right)$ given by

$$
\begin{aligned}
& y_{n}=b_{n}-y \quad \text { if } \quad n<S(1) \\
& y_{n}=v_{n, k} \quad \text { si } \quad n \in[S(k), S(k+1)]
\end{aligned}
$$

converges to zero, the sequence $b_{n}=y_{n}+y$ converges to $y$, and according to relation (4.6) we have

$$
b_{n} \leq_{c} f\left(x_{n}\right) \quad \forall n \geq 1 .
$$

Consequently, $y \in s-A_{\bar{x}}^{f}$.

In a similar way, we show that if $E$ and $F$ are metrizable, then :

$$
s-B_{\bar{x}}^{f}=B_{\bar{x}}^{f},
$$


the proof is complete.

Remark 4.3. In what follows, we assume that $E$ and $F$ are metrizable and adopt the notation $A_{\bar{x}}^{f}$ for a lower level set.

Next, we prove the following elementary property.

Proposition 4.4. Let $f: E \rightarrow F^{\bullet}$ and $\bar{x} \in \operatorname{Dom} f$. Then,

(1) $A_{\bar{x}}^{f}=A_{\bar{x}}^{f}-C$;

(2) $B_{\bar{x}}^{f}=B_{\bar{x}}^{f}+C$.

Proof. 1) Let $y \in A_{\bar{x}}^{f}, c \in C,\left(x_{n}\right)$ be a sequence in $E$ converging to $\bar{x}$. There exists a sequence $\left(b_{n}\right)$ in $F$ such that

$$
\lim _{n \rightarrow+\infty} b_{n}=y \quad \text { and } \quad b_{n} \leq_{c} f\left(x_{n}\right), \quad \forall n \in \mathbb{N} .
$$

Set $a_{n}=b_{n}-c$ and remark that

$$
\lim _{n \rightarrow+\infty} a_{n}=y-c .
$$

As $f\left(x_{n}\right)-b_{n} \in C \cup\{+\infty\}$ and $c \in C$ we have

$$
f\left(x_{n}\right)-b_{n}+c \in C \cup\{+\infty\}
$$

for every $n \in \mathbb{N}$. Consequently

$$
a_{n} \leq_{c} f\left(x_{n}\right), \quad \forall n \in \mathbb{N} .
$$

Using (4.7) and (4.8), we deduce that $y-c \in A_{\bar{x}}^{f}$ for every $c \in C$ and $y \in A_{\bar{x}}^{f}$, whence

$$
A_{\bar{x}}^{f}-C \subset A_{\bar{x}}^{f}
$$

The other inclusion is true because $0 \in C$.

2) The second equality can be established by inverting the order.

Theorem 4.5. Let $f: E \rightarrow F^{\bullet}$ and $\bar{x} \in \operatorname{Dom} f$. Then,

(1) $f$ is l.s.c at $\bar{x} \Longleftrightarrow A_{\bar{x}}^{f}=f(\bar{x})-C$;

(2) $f$ is u.s.c at $\bar{x} \Longleftrightarrow B_{\bar{x}}^{f}=f(\bar{x})+C$.

Proof. $(\Rightarrow)$

Suppose that $f$ is l.s.c at $\bar{x}$ and $y \in A_{\bar{x}}^{f}$. We can easily see that (as detailed in Proposition 5.1),

$$
y-f(\bar{x}) \in-C .
$$

Therefore

$$
A_{\bar{x}}^{f} \subset f(\bar{x})-C .
$$


Since $f$ is l.s.c at $\bar{x}, f(\bar{x}) \in A_{\bar{x}}^{f}$, by Proposition 4.4, we have

$$
f(\bar{x})-C \subset A_{\bar{x}}^{f} .
$$

We hence deduce, via (4.9) and (4.10), that

$$
f(\bar{x})-C=A_{\bar{x}}^{f} .
$$

$(\Leftarrow)$ Assume that $A_{\bar{x}}^{f}=f(\bar{x})-C$. As $0 \in C, f(\bar{x}) \in A_{\bar{x}}^{f}$. Following Proposition 4.1, $f$ is lower semicontinuous at $\bar{x}$.

2) The second equivalence can be established similarly.

Corollary 4.6. Let $f: E \rightarrow F^{\bullet}$ and $\bar{x} \in \operatorname{Dom} f$. Assume that $C$ is pointed and $(F, C)$ is normal. Then, the assertions below are equivalent.

i) $f$ is continuous at $\bar{x}$;

ii) $A_{\bar{x}}^{f} \cap B_{\bar{x}}^{f} \neq \emptyset$.

Proof. ii) $\Rightarrow$ i).

Let $y \in A_{\bar{x}}^{f} \cap B_{\bar{x}}^{f}$. Then, for each sequence $\left(x_{n}\right)$ that converges to $\bar{x}$, there exist two sequences $\left(a_{n}\right)$ and $\left(b_{n}\right)$ in $F$ such that

$$
\lim _{n \rightarrow+\infty} a_{n}=y \text { and } a_{n} \leq_{c} f\left(x_{n}\right), \forall n \in \mathbb{N},
$$

and

$$
\lim _{n \rightarrow+\infty} b_{n}=y \text { and } f\left(x_{n}\right) \leq_{c} b_{n}, \quad \forall n \in \mathbb{N} .
$$

In particular, for the stationary sequence $x_{n}=x$, for every $n \in \mathbb{N}$, there exists two sequences $\left(a_{n}^{\prime}\right)$ and $\left(b_{n}^{\prime}\right)$ in $F$ such that

$$
\lim _{n \rightarrow+\infty} a_{n}^{\prime}=y \text { and } a_{n}^{\prime} \leq_{c} f(\bar{x}), \quad \forall n \in \mathbb{N}
$$

and

$$
\lim _{n \rightarrow+\infty} b_{n}^{\prime}=y \text { and } f(\bar{x}) \leq_{c} b_{n}^{\prime}, \quad \forall n \in \mathbb{N} .
$$

Since $C$ is closed,

$$
f(\bar{x})-y \in C \cap(-C) .
$$

Since $C$ is pointed, then $f(\bar{x})=y \in A_{\bar{x}}^{f} \cap B_{\bar{x}}^{f}$. Following Remark 4.1, $f$ is l.s.c and u.s.c at $\bar{x}$. The normality of $(F, C)$ ensures the continuity of $f$ at $\bar{x}$. 


\section{Properties of LOWER AND UPPER LEVEL SETS}

This section is devoted to the study of the behavior of the lower and upper level sets. We show that they have remarkable properties, both topological and geometrical. We focus only on the lower level set, because the properties of the local upper level set can be deduced straightforwardly by similarity.

At first, we provide a bound for $A_{\bar{x}}^{f}$ and $B_{\bar{x}}^{f}$.

Proposition 5.1. Let $f: E \rightarrow F^{\bullet}$ and $\bar{x} \in \operatorname{Dom} f$. The following assertion hold:

$$
A_{\bar{x}}^{f} \leq_{c}\{f(\bar{x})\} \leq_{c} B_{\bar{x}}^{f} .
$$

Proof. Let $y \in A_{\bar{x}}^{f}$ and consider the sequence $\left(x_{n}\right)$ given by $x_{n}=\bar{x}$ for every $n \in \mathbb{N}$. There exists a sequence $\left(b_{n}\right)$ in $F$ such that

$$
\lim _{n \rightarrow+\infty} b_{n}=y \quad \text { and } \quad b_{n} \leq_{c} f(\bar{x}), \quad \forall n \in \mathbb{N} .
$$

Since $C$ is closed, $y \leq_{c} f(\bar{x})$. Hence, $A_{\bar{x}}^{f} \leq_{c}\{f(\bar{x})\}$.

In the same manner, we show that $\{f(\bar{x})\} \leq_{c} \quad B_{\bar{x}}^{f}$ by inverting the order.

The useful property of directness is also verified, precisely we have the following:

Proposition 5.2. Let $f: E \rightarrow F^{\bullet}$ and $\bar{x} \in \operatorname{Dom} f$. Suppose that $F$ is a Banach lattice. Then, $A_{\bar{x}}^{f}$ is directed upwards and $B_{\bar{x}}^{f}$ is directed downwards.

Proof. Take $y_{1}, y_{2} \in A_{\bar{x}}^{f}$ and $\left(x_{n}\right)$ a sequence in $E$ converging to $\bar{x}$. Then there exist two sequences $\left(b_{n}\right)$ and $\left(b_{n}^{\prime}\right)$ in $F$ such that

$$
\lim _{n \rightarrow+\infty} b_{n}=y_{1} \quad \text { and } \quad b_{n} \leq_{c} f\left(x_{n}\right), \quad \forall n \in \mathbb{N}
$$

and

$$
\lim _{n \rightarrow+\infty} b_{n}^{\prime}=y_{2} \quad \text { and } \quad b_{n}^{\prime} \leq_{c} f\left(x_{n}\right), \quad \forall n \in \mathbb{N} .
$$

As the map :

$$
\begin{aligned}
\sup : \quad F \times F & \rightarrow F \\
(x, y) & \mapsto \sup (x, y)
\end{aligned}
$$

is uniformly continuous (see [20] Proposition 5.2 page 83), taking into consideration (5.1) and (5.2), we obtain

$$
\lim _{n \rightarrow+\infty} \sup \left(b_{n}, b_{n}^{\prime}\right)=\sup \left(y_{1}, y_{2}\right) \quad \text { and } \sup \left(b_{n}, b_{n}^{\prime}\right) \leq_{c} f\left(x_{n}\right), \quad \forall n \in \mathbb{N} .
$$

It follows that $\sup \left(y_{1}, y_{2}\right) \in A_{\bar{x}}^{f}$. 
Proposition 5.3. For each $\xi \in Y^{*}, \xi\left(\sup A_{\bar{x}}^{f}\right)=\sup \xi\left(A_{\bar{x}}^{f}\right)$.

Proof. Let $\xi \in Y^{*}$. $A_{\bar{x}}^{f}$ being directed upwards, thanks to Proposition 4.1 of [2], it suffices to check that $\xi$ is continuous with respect to the Scott topology. Since $\mathbb{R}$ is continuous (in sense of [2] see example 1.1 in [2] for further details) and $\xi$ is lower semicontinuous continuous (because continuous), it follows from [Theorem 4.2, [2]] that $\xi$ is Scottcontinuous. The proof is then complete.

Proposition 5.4. Let $f: E \rightarrow F^{\bullet}$ and $\bar{x} \in \operatorname{Dom} f$. Then $A_{\bar{x}}^{f}$ and $B_{\bar{x}}^{f}$ are convex.

Proof. Take $y_{1}, y_{2} \in A_{\bar{x}}^{f}, \lambda \in[0,1]$ and $\left(x_{n}\right)$ a sequence in $E$ that converges to $\bar{x}$, then there exist two sequences $\left(b_{n}^{\prime}\right)$ and $\left(b_{n}^{\prime \prime}\right)$ in $F$ such that

$$
\lim _{n \rightarrow+\infty} b_{n}^{\prime}=y_{1} \quad \text { and } \quad b_{n}^{\prime} \leq_{c} f\left(x_{n}\right), \quad \forall n \in \mathbb{N},
$$

and

$$
\lim _{n \rightarrow+\infty} b_{n}^{\prime \prime}=y_{2} \quad \text { and } \quad b_{n}^{\prime \prime} \leq_{c} f\left(x_{n}\right), \quad \forall n \in \mathbb{N} .
$$

Take $b_{n}=\lambda b_{n}^{\prime}+(1-\lambda) b_{n}^{\prime \prime}$. On one hand we have

$$
\lim _{n \rightarrow+\infty} b_{n}=\lambda y_{1}+(1-\lambda) y_{2} \text {. }
$$

On the other hand,

$$
\begin{aligned}
f\left(x_{n}\right)-b_{n} & =f\left(x_{n}\right)-\lambda b_{n}^{\prime}-(1-\lambda) b_{n}^{\prime \prime} \\
& =\lambda\left(f\left(x_{n}\right)-b_{n}^{\prime}\right)+(1-\lambda)\left(f\left(x_{n}\right)-b_{n}^{\prime \prime}\right) .
\end{aligned}
$$

As $f\left(x_{n}\right)-b_{n}^{\prime} \in C \cup\{+\infty\}$ and $f\left(x_{n}\right)-b_{n}^{\prime \prime} \in C \cup\{+\infty\}$,

$$
b_{n} \leq_{c} f\left(x_{n}\right), \quad \forall n \in \mathbb{N}
$$

we deduce from (5.3) and (5.4) that

$$
\lambda y_{1}+(1-\lambda) y_{2} \in A_{\bar{x}}^{f}
$$

Similarly, we can prove that $B_{\bar{x}}^{f}$ is convex.

\section{THE OBJECTIVE OF THE PAPER}

The study of the l.s.c regularization of vector-valued mappings has been initiated by Théra for maps with values in complete (lattice) Daniell spaces. Our ambition here is to define a l.s.c of a vector-valued mapping $f: E \rightarrow F^{\bullet}$, when $F$ is a complete Banach lattice ordered by a closed convex cone $C$ with nonempty interior. 
Assuming that for every $\bar{x} \in \operatorname{Dom} f, A_{\bar{x}}^{f} \neq \varnothing$, let us agree to introduce the following mapping:

$$
I_{f}(\bar{x}):=\sup A_{\bar{x}}^{f} .
$$

Remark 6.1. $F$ being complete lattice, $A_{\bar{x}}^{f}$ is upper bounded, it follows that $I_{f}$ is well defined i.e., $\sup A_{\bar{x}}^{f}$ exists.

We first begin with the case where $F=H$ is a separable complete Hilbert lattice space. Let $\left(e_{n}\right)_{n \in \mathbb{N}}$ be a basis of $H$. The order on $H$ is given by the closed convex cone given by

$$
H_{+}=\left\{x \in H \mid\left\langle e_{p}, x\right\rangle \geq 0, \forall p \in \mathbb{N}\right\} .
$$

Clearly, the polar cone of $H_{+}$is equal to $H_{+}$, i.e. , $H_{+}^{*}=H_{+}$.

In order to conclude the semicontinuity of $I_{f}$ in this first case with the use of semicontinuity of the usual scalar lower limit, we need two ingredients.

Lemma 6.2. Let $f: E \rightarrow H^{\bullet}$ and $\bar{x} \in \operatorname{Dom} f$. Assume that $A_{\bar{x}}^{f} \neq \emptyset$. Then, for each $p \in \mathbb{N}$, one has

$$
\left\langle e_{p}, A_{\bar{x}}^{f}\right\rangle=A_{\bar{x}}^{\left\langle e_{p}, f\right\rangle}
$$

where $\left\langle e_{p}, A_{\bar{x}}^{f}\right\rangle:=\left\{\left\langle e_{p}, y\right\rangle \mid y \in A_{\bar{x}}^{f}\right\}$.

Proof. Let $p \in \mathbb{N}, y \in A_{\bar{x}}^{f}$ and $\left(x_{n}\right)$ be a sequence in $E$ converging to $\bar{x}$, then there is a sequence $\left(b_{n}\right)$ in $H$ such that

$$
\lim _{n \rightarrow+\infty} b_{n}=y \quad \text { and } \quad b_{n} \leq f\left(x_{n}\right), \quad \forall n \in \mathbb{N},
$$

which implies that

$$
\lim _{n \rightarrow+\infty}\left\langle e_{p}, b_{n}\right\rangle=\left\langle e_{p}, y\right\rangle \quad \text { and } \quad\left\langle e_{p}, b_{n}\right\rangle \leq\left\langle e_{p}, f\left(x_{n}\right)\right\rangle, \quad \forall n \in \mathbb{N}
$$

and therefore $\left\langle e_{p}, y\right\rangle \in A_{\bar{x}}^{\left\langle e_{p}, f\right\rangle}$. It follows that

$$
\left\langle e_{p}, A_{\bar{x}}^{f}\right\rangle \subset A_{\bar{x}}^{\left\langle e_{p}, f\right\rangle} \text { for each } p \in \mathbb{N} \text {. }
$$

At this stage, let us show that

$$
A_{\bar{x}}^{\left\langle e_{p}, f\right\rangle} \subset\left\langle e_{p}, A_{\bar{x}}^{f}\right\rangle
$$

for each $p \in \mathbb{N}$.

So, let $\left(x_{n}\right)$ be a sequence in $E$ converging to $\bar{x}, p \in \mathbb{N}$ and $y \in A_{\bar{x}}^{\left\langle e_{p}, f\right\rangle}$. Then consider $\left(y_{n}\right)$ in $\mathbb{R}$ such that

$$
\lim _{n \rightarrow+\infty} y_{n}=y \quad \text { and } \quad y_{n} \leq\left\langle e_{p}, f\left(x_{n}\right)\right\rangle, \quad \forall n \in \mathbb{N} .
$$


Let $z_{0} \in A_{\bar{x}}^{f}$ be fixed and take $z:=z_{0}+\left(y-z_{0}^{p}\right) e_{p}$, where $z_{0}^{p}=\left\langle z_{0}, e_{p}\right\rangle$. Let us show that $z \in A_{\bar{x}}^{f}$.

As $z_{0} \in A_{\bar{x}}^{f}$, there exists $\left(z_{n}\right)$ such that

$$
\lim _{n \rightarrow+\infty} z_{n}=z_{0} \quad \text { and } \quad z_{n} \leq f\left(x_{n}\right), \quad \forall n \in \mathbb{N} .
$$

Set $z_{n}^{\prime}=z_{n}+\left(y_{n}-z_{n}^{p}\right) e_{p}$ with $z_{n}^{p}=\left\langle z_{n}, e_{p}\right\rangle$. Since $y \in A_{\bar{x}}^{\left\langle e_{p}, f\right\rangle}$, according to (6.2) we have

$$
\lim _{n \rightarrow+\infty} z_{n}^{\prime}=z \quad \text { and } \quad z_{n}^{\prime} \leq f\left(x_{n}\right), \quad \forall n \in \mathbb{N}
$$

with $\left\langle e_{p}, z\right\rangle=y$. We deduce that

$$
A_{\bar{x}}^{\left\langle e_{p}, f\right\rangle} \subset\left\langle e_{p}, A_{\bar{x}}^{f}\right\rangle .
$$

Using (6.1) and (6.3) we obtain $A_{\bar{x}}^{\left\langle e_{p}, f\right\rangle}=\left\langle e_{p}, A_{\bar{x}}^{f}\right\rangle \quad$ for each $p \in \mathbb{N}$.

Lemma 6.3. [17] Let $Y=\prod_{i} Y_{i}$ be the space product of a family of ordered vector spaces $Y_{i}$. Then, a map $f: E \rightarrow Y$ is l.s.c, if and only if, its projections $f_{i}=p_{i} \circ f$ are l.s.c.

Here, $p_{i}$ denotes the projection from $Y$ into $Y_{i}$.

Theorem 6.4. Let $f: E \rightarrow H^{\bullet}$ and let $\bar{x} \in \operatorname{Dom} f$. Then, $I_{f}$ is l.s.c at $\bar{x}$.

Proof. According to Proposition 5.3, for all $p \geq 0$ we have

$$
\left\langle e_{p}, I_{f}(\bar{x})\right\rangle=\left\langle e_{p}, \sup A_{\bar{x}}^{f}\right\rangle=\sup \left\langle e_{p}, A_{\bar{x}}^{f}\right\rangle .
$$

Then, using Lemma 6.2 and Remark 3.3, we deduce that

$$
\left\langle e_{p}, I_{f}(\bar{x})\right\rangle=\sup A_{\bar{x}}^{f_{p}}=\liminf _{x \rightarrow \bar{x}} f_{p}(x),
$$

where $f_{p}=\left\langle e_{p}, f\right\rangle$. Clearly, $\left\langle e_{p}, I_{f}\right\rangle$ is l.s.c for each $p$, thus $f$ is l.s.c.

Next, we present the more general case where $F$ is a complete Banach lattice in which we do not make recourse to semicontinuity of the scalar lower limit.

Theorem 6.5. Suppose $F$ is a complete Banach lattice. Then, $I_{f}$ is lower semicontinuous at every $x$ in $\operatorname{Dom} f$, and therefore $I_{f}$ defines a l.s.c regularization of $f$.

We prove first some technical Lemmata that will be useful for proving the main result. 
Lemma 6.6. For every convex cone $C$ in $F$, we have

$$
(F \backslash C)-C=F \backslash C .
$$

Proof. The proof is standard and based on the convexity of $C$.

Lemma 6.7. We have $I_{f}(\bar{x}) \in \operatorname{cl} A_{\bar{x}}^{f}$.

Proof. It suffices to prove for each $\mu \in \operatorname{Int} C$ that

$$
I_{f}(\bar{x})-\mu \in \operatorname{cl} A_{\bar{x}}^{f} .
$$

To this end, we shall prove that for every $y \in F$ such that

$$
I_{f}(\bar{x})-y \in \operatorname{Int} C
$$

then $y \in \operatorname{cl} A_{\bar{x}}^{f}$.

Assume on the contrary that there exists $y$ such that

$$
I_{f}(\bar{x})-y \in \operatorname{Int} C \text { and } y \notin \operatorname{cl} A_{\bar{x}}^{f} .
$$

As $A_{\bar{x}}^{f}=A_{\bar{x}}^{f}-C$ and $C$ is closed, it follows that

$$
\operatorname{cl}\left(A_{\bar{x}}^{f}\right)-C \subset \operatorname{cl}\left(A_{\bar{x}}^{f}-C\right)=\operatorname{cl} A_{\bar{x}}^{f}
$$

therefore $0 \notin y-\operatorname{cl}\left(A_{\bar{x}}^{f}\right)+C$. We see that $y-\operatorname{cl}\left(A_{\bar{x}}^{f}\right)+C \neq \emptyset$ and $y-A_{\bar{x}}^{f}+C=y-A_{\bar{x}}^{f}$ is convex (because $A_{\bar{x}}^{f}$ is convex)

According to the Hahn-Banach Separation Theorem, select some $y^{*} \in$ $F^{*}$ such that $y^{*} \neq 0$ and

$$
\left\langle y^{*}, y-z+r\right\rangle>0
$$

for each $z \in \operatorname{cl} A_{\bar{x}}^{f}$ and $r \in C$.

Let us show that $y^{*} \in C_{+}^{*} \backslash\{0\}$. Let $r \in C$ and let $z \in \operatorname{cl} A_{\bar{x}}^{f}$. Thanks to $(6.5)$, we deduce

$$
\left\langle y^{*}, y-z\right\rangle>-\left\langle y^{*}, n r\right\rangle
$$

for each $n \in \mathbb{N}$, and therefore

$$
\frac{1}{n}\left\langle y^{*}, y-z\right\rangle>-\left\langle y^{*}, r\right\rangle \text {. }
$$

Letting $n$ go to $+\infty$, we obtain $\left\langle y^{*}, r\right\rangle \geq 0$ for $r \in C$. Thus,

$$
y^{*} \in C^{*} \backslash\{0\} .
$$

Now, let $r \in C$. We have $\frac{1}{n} r \in C$, consequently

$$
\left\langle y^{*}, y-z+\frac{1}{n} r\right\rangle \geq 0
$$

by passing to the limit whenever $n$ goes to $+\infty$, we obtain

$$
\left\langle y^{*}, y\right\rangle \geq\left\langle y^{*}, z\right\rangle \text { for each } z \in A_{\bar{x}}^{f} \text {. }
$$


Therefore,

$$
\left\langle y^{*}, y\right\rangle \geq \sup _{z \in A_{\bar{x}}^{f}}\left\langle y^{*}, z\right\rangle .
$$

Since $A_{\bar{x}}^{f}$ is directed upwards, it follows from Proposition 5.3 that

$$
\left\langle y^{*}, I_{f}(\bar{x})\right\rangle=\sup _{y \in A_{\bar{x}}^{f}}\left\langle y^{*}, y\right\rangle \text {. }
$$

Accordingly,

$$
\left\langle y^{*}, y\right\rangle \geq\left\langle y^{*}, \sup _{z \in A_{\bar{x}}^{f}} z\right\rangle .
$$

Keeping in mind that $I_{f}(\bar{x})-y \in \operatorname{Int} C$ and $y^{*} \in C_{+}^{*} \backslash\{0\}$, thanks to Remark 2.1 we conclude that

$$
\left\langle y^{*}, \sup A_{\bar{x}}^{f}-y\right\rangle>0,
$$

and consequently

$$
\left\langle y^{*}, I_{f}(\bar{x})\right\rangle>\left\langle y^{*}, y\right\rangle
$$

Relations (6.6) and (6.7) provide the contradiction.

Then, for each $y \in F$ such $I_{f}(\bar{x})-y \in \operatorname{Int} C$, we have

$$
y \in \operatorname{cl} A_{\bar{x}}^{f} .
$$

In particular, taking $y=I_{f}(\bar{x})-\mu(\mu \in \operatorname{Int} C)$, We derive that

$$
I_{f}(\bar{x})-y=\mu \in \operatorname{Int} C,
$$

we therefore deduce that

$$
I_{f}(\bar{x})-\mu \in \operatorname{cl} A_{\bar{x}}^{f}
$$

$\mu$ being arbitrary in $\operatorname{Int} C$, we end up at

$$
I_{f}(\bar{x}) \in \operatorname{cl} A_{\bar{x}}^{f} .
$$

As a consequence of the previous Lemma we derive the following technical Lemma.

Lemma 6.8. For each $\bar{y} \in A_{\bar{x}}^{f}$ such that $\bar{y}<_{c} I_{f}(\bar{x})$, there exists a sequence $\left(\beta_{k}\right)_{k}$ in $\operatorname{cl} A_{\bar{x}}^{f}$ such that

(6.8) $\beta_{k} \rightarrow I_{f}(\bar{x})$ as $n$ goes to $+\infty$ and $\bar{y}<_{c} \beta_{k}$, for all $k$. 
Proof. For $k>0$, take

$$
\beta_{k}=I_{f}(\bar{x})-\frac{1}{k}\left(I_{f}(\bar{x})-\bar{y}\right) .
$$

Observe that

$$
\beta_{k}-\bar{y}=\frac{k-1}{k}\left(I_{f}(\bar{x})-\bar{y}\right) \in \operatorname{Int} C
$$

and conclude that

$$
\bar{y}<_{c} \beta_{k}, \forall k .
$$

On the other hand, Lemma 6.7 implies

$$
I_{f}(\bar{x}) \in \operatorname{cl} A_{\bar{x}}^{f} .
$$

Then, (6.9) leads to

$$
\beta_{k} \in \operatorname{cl}\left(A_{\bar{x}}^{f}\right)-C \subset \operatorname{cl}\left(A_{\bar{x}}^{f}-C\right)=\operatorname{cl} A_{\bar{x}}^{f} .
$$

Clearly, $\left(\beta_{k}\right)$ converges to $I_{f}(\bar{x})$. The proof of the Lemma is therefore complete.

Now, for $\bar{x} \in \operatorname{Dom} f$, we introduce the following sets

$$
E_{f}(\bar{x}):=\left\{y \in A_{\bar{x}}^{f} \mid y<_{c} I_{f}(\bar{x})\right\}
$$

and

$$
H_{f}(\bar{x}):=\left\{y \in \operatorname{cl} A_{\bar{x}}^{f} \mid y \leq_{c} I_{f}(\bar{x})\right\}
$$

and establish the following:

Lemma 6.9. We have $c l E_{f}(\bar{x})=H_{f}(\bar{x})$.

Proof. Let $y \in \operatorname{cl}\left(E_{f}(\bar{x})\right)$. There exists a sequence $\left(y_{k}\right)$ converging to $y$ such that $y_{k} \in E_{f}(\bar{x})$, for all $k$. Clearly, $y \in \operatorname{cl} A_{\bar{x}}^{f}$ and

$$
I_{f}(\bar{x})-y_{k} \in \operatorname{Int} C \subset C \forall k .
$$

Passing to the limit in (6.12) whenever $k$ goes to $+\infty$, we obtain that

$$
I_{f}(\bar{x})-y \in C,
$$

or equivalently $y \in H_{f}(\bar{x})$.

Conversely, let $\bar{y} \in H_{f}(\bar{x})$. Then, $\bar{y} \in \operatorname{cl} A_{\bar{x}}^{f}$ and $I_{f}(\bar{x})-\bar{y} \in C$. Therefore, there exists a sequence $\left(y_{k}\right)$ in $A_{\bar{x}}^{f}$ such that $y_{k} \rightarrow \bar{y}$. Consider now a sequence $\left(\nu_{k}\right)$ in Int $C$ such that $\nu_{k} \rightarrow 0$. Take $\bar{y}_{k}:=y_{k}-\nu_{k}$ and observe that $\left(\bar{y}_{k}\right)_{k}$ also converges to $\bar{y}$. On the other hand, Proposition 4.4 implies that

$$
\bar{y}_{k} \in A_{\bar{x}}^{f}-\operatorname{Int} C \subset A_{\bar{x}}^{f}-C=A_{\bar{x}}^{f} .
$$


Remark also that

$$
\begin{aligned}
I_{f}(\bar{x})-\bar{y}_{k} & =\left(I_{f}(\bar{x})-y_{k}\right)+\nu_{k} \\
& \in C+\operatorname{Int} C=\operatorname{Int} C .
\end{aligned}
$$

Thus, $\bar{y} \in \operatorname{cl} E_{f}(\bar{x})$. The proof of the Lemma is therefore established.

Remark 6.10. Remark that $A_{\bar{x}}^{f} \subset \operatorname{cl} E_{f}(\bar{x})=H_{f}(\bar{x})=\operatorname{cl} A_{\bar{x}}^{f}$.

The following result will play a key role to derive the lower semicontinuity of the lower limit.

Lemma 6.11. For every sequence $\left(x_{k}\right)_{k}$ converging to $\bar{x}$, the sequence $\left(A_{x_{k}}^{f}\right)_{k}$ is lower convergent to $A_{\bar{x}}^{f}$ in the sense of Painlevé-Kuratowski.

Proof. Let us first observe that, thanks to Remark 6.10, it suffices to prove that

$$
E_{f}(\bar{x}) \subset \liminf _{k} A_{x_{k}}^{f} .
$$

Let $\bar{y} \in E_{f}(\bar{x})$. We shall prove that

$$
\bar{y} \in \liminf _{k} A_{x_{k}}^{f} .
$$

Obviously, $\bar{y}<_{c} I_{f}(\bar{x})$. Let $\left(\beta_{k}\right) \in \mathrm{cl} A_{\bar{x}}^{f}$ be the sequence provided by Lemma 6.8 such that $\beta_{k} \rightarrow I_{f}(\bar{x})$ and

$$
\bar{y}<_{c} \beta_{k}, \forall k>0 \text {. }
$$

Consider now $y_{k} \rightarrow \bar{y}$ with $y_{k}<_{c} \bar{y}$ for all $k \geq 0$. We claim that

$$
\exists \delta_{0}>0: \forall k>0, y_{k} \leq_{c} f(x) \forall x \in B\left(\bar{x}, \delta_{0}\right) .
$$

Otherwise, for each $\delta>0$, there exists $k>0$ and $x_{\delta} \in B(\bar{x}, \delta)$ such that

$$
f\left(x_{\delta}\right)-y_{k} \notin C .
$$

Thus,

$$
\begin{aligned}
f\left(x_{\delta}\right)-\bar{y} & \in y_{k}-\bar{y}+F \backslash C \\
& \in-C+F \backslash C=F \backslash C .
\end{aligned}
$$

Therefore, we can exhibit a sequence $\left(w_{n}\right)$ converging to $\bar{x}$ such that

$$
f\left(w_{n}\right)-\bar{y} \in F \backslash C, \forall n .
$$

Now, consider some $k_{0}>0$. Since $\beta_{k_{0}} \in \operatorname{cl} A_{\bar{x}}^{f}$, there exists a sequence $\left(\gamma_{m}\right)=\left(\gamma_{m}\left(k_{0}\right)\right)_{m}$ such that

$$
\gamma_{m} \rightarrow \beta_{k_{0}}
$$


with $\gamma_{m} \in A_{\bar{x}}^{f}$. Thus, from the definition of the lower level set, there exists a sequence $\left(\rho_{n}\right)_{n}:=\left(\rho_{n}\left(k_{0}, m\right)\right)_{n}$ converging to $\gamma_{m}$ and satisfying for each $n$

$$
f\left(w_{n}\right)-\rho_{n} \in C, \text { or else } \rho_{n}-f\left(w_{n}\right) \in-C .
$$

Hence, by (6.17), (6.18) and Lemma 6.6, we obtain

$$
\rho_{n}-\bar{y} \in(F \backslash C)-C=F \backslash C .
$$

Therefore

$$
\rho_{n}-\bar{y} \notin \operatorname{Int} C .
$$

Passing to limit in (6.20) whenever $n$ goes to $+\infty$ we obtain

$$
\gamma_{m}-\bar{y} \notin \operatorname{Int} C,
$$

and thus, by passing to limit in (6.21) whenever $m$ goes to $+\infty$, we obtain

$$
\beta_{k_{0}}-\bar{y} \notin \operatorname{Int} C
$$

contradicting (6.15). Then (6.16) is true.

Now, using (6.16), let us prove that $y_{k} \in A_{x_{k}}^{f}$. To this end, let $\left(x_{k}^{n}\right)_{n} \rightarrow x_{k}$. For some $n_{0}:=n_{0}(k)$, we have $x_{k}^{n} \in B\left(x_{k}, \frac{\delta_{0}}{2}\right) \forall n \geq n_{0}$. Let $\left(z_{k}^{n}\right)_{n}$ be a sequence converging to $y_{k}$ such that for each $k$

$$
z_{k}^{n} \leq_{c} y_{k} .
$$

Take $y_{k}^{n}=z_{k}^{n}$ for $n \geq n_{0}$ and $y_{k}^{n}=f\left(x_{k}^{n}\right)$ for $n<n_{0}$.

We can suppose that $x_{k} \in B\left(\bar{x}, \frac{\delta_{0}}{2}\right)$ and we note that $x_{k}^{n} \in B\left(\bar{x}, \delta_{0}\right)$. Hence, thanks to (6.16), we see that

$$
y_{k}^{n} \leq_{c} f\left(x_{k}^{n}\right) \forall n,
$$

Consequently, $y_{k} \in A_{x_{k}}^{f}$ for all $k$. Thus, $\bar{y} \in \liminf _{k} A_{x_{k}}^{f}$. Hence,

$$
E_{f}(\bar{x}) \subset \liminf _{k} A_{x_{k}}^{f} .
$$

Since $\liminf _{k} A_{x_{k}}^{f}$ is closed, it follows that

$$
\operatorname{cl}\left(E_{f}(\bar{x})\right) \subset \liminf _{k} A_{x_{k}}^{f} .
$$

Then, Remark 6.10 implies that

$$
A_{\bar{x}}^{f} \subset \liminf _{k} A_{x_{k}}^{f} .
$$

The proof is therefore complete.

We are now ready to conclude the main result of the paper. 
Proof. Let $\bar{x} \in \operatorname{Dom} f$ and let $\left(x_{n}\right)$ be a sequence converging to $\bar{x}$. Thanks to Lemma 6.7, there exists a sequence $\left(y_{k}\right)$ converging to $I_{f}(\bar{x})$ such that $y_{k} \in A_{\bar{x}}^{f}$. From Lemma 6.11, it follows that $y_{k} \in \liminf _{n} A_{x_{n}}^{f}$. Select $y_{k}^{n} \in A_{x_{n}}^{f}$ such that $\left(y_{k}^{n}\right)_{n}$ converges to $y_{k}$ as $n$ goes to $+\infty$. Following the beginning of the proof of Lemma 6.11 we can assume that $\left(y_{k}^{n}\right)$ converges uniformly in $k$ to $y_{k}$. Indeed, we can consider $y_{k}^{n}=y_{k}-\nu_{n}$ with $\nu_{n} \in \operatorname{Int} C$ such that

$$
\lim _{n \rightarrow+\infty} \nu_{n}=0 \text {. }
$$

Clearly,

$$
y_{k}^{n} \leq_{c} \sup A_{x_{n}}^{f}=I_{f}\left(x_{n}\right) .
$$

This being true for all $k$, let $k(n)$ be a map such that $k(n) \rightarrow+\infty$. Take $b_{n}=y_{k(n)}^{n}$; this sequence converges to $I_{f}(\bar{x})$ (thanks to the uniform convergence of $y_{k}^{n}$ to $y_{k}$ ) and satisfies

$$
b_{n} \leq_{c} I_{f}\left(x_{n}\right)
$$

The proof is complete.

Remark 6.12. From now on, we will use the following notation

$$
v-\liminf _{x \rightarrow \bar{x}} f(x):=I_{f}(\bar{x})=\sup A_{\bar{x}}^{f}
$$

Corollary 6.13. The assertions below are equivalent

- $f$ is lower semicontinuous at $\bar{x} \in \operatorname{Dom} f$;

- $f(\bar{x}) \leq_{c} v-\liminf _{x \rightarrow \bar{x}} f(x)$.

Proof. By Proposition 4.1, $f$ is l.s.c at $\bar{x}$ if, and only if $f(\bar{x}) \in A_{\bar{x}}^{f}$. Then, if $f$ is l.s.c at $\bar{x}$, as $v-\liminf _{x \rightarrow \bar{x}} f(x)$ is the least upper bound of $A_{\bar{x}}^{f}$, it follows that

$$
f(\bar{x}) \leq_{c} v-\liminf _{x \rightarrow \bar{x}} f(x) .
$$

Conversely, suppose that (6.25) holds. We know that $f(\bar{x})$ is an upper bound of $A_{\bar{x}}^{f}$, a fortiori

$$
v-\liminf _{x \rightarrow \bar{x}} f(x) \leq_{c} f(\bar{x}) .
$$

Hence, as the cone $C$ is pointed, we derive

$$
v-\liminf _{x \rightarrow \bar{x}} f(x)=f(\bar{x}) .
$$


Therefore, thanks to the characterization of semicontinuity of [4] and our main result, we deduce that $f$ is necessarily l.s.c at $\bar{x}$. Indeed, having in mind

$$
I_{f}(z):=v-\liminf _{x \rightarrow z} f(x) \leq_{c} f(z), \forall z \in E,
$$

for any sequence $\left(x_{n}\right)$ such that $x_{n} \rightarrow \bar{x}$, by Theorem 6.5 , there exists a sequence $\left(b_{n}\right)$ converging to $I_{f}(\bar{x})=f(\bar{x})$ such that

$$
b_{n} \leq_{c} I_{f}\left(x_{n}\right) \leq_{c} f\left(x_{n}\right) \forall n .
$$

The proof is established.

Remark 6.14. Similarly, we can define the upper semicontinuous regularization of $f$ by

$$
v-\limsup _{x \rightarrow \bar{x}} f(x):=\inf B_{\bar{x}}^{f}
$$

\section{Compatibility With the Standard CASES}

Now, we derive from the main result a more flexible proof for the well know result that says : for every real-valued function, the lower limit is lower semicontinuous. We consider here, more generally, finite dimensional-valued mappings.

Consider $F=\mathbb{R}^{p}, f=\left(f_{1}, f_{2}, \ldots, f_{p}\right)$ and $\bar{x} \in \operatorname{Dom} f$. Note that the order in this case goes back to the usual order of $\mathbb{R}$, generated by the cone $C:=\mathbb{R}_{+}^{p}$ and will be simply denoted by $\leq$.

We claim that

$$
\left.\left.\operatorname{cl} A_{\bar{x}}^{f}=\prod_{i=1}^{p}\right]-\infty, \liminf _{x \rightarrow \bar{x}} f_{i}(x)\right]
$$

and

$$
\operatorname{cl} B_{\bar{x}}^{f}=\prod_{i=1}^{p}\left[\limsup _{x \rightarrow \bar{x}} f_{i}(x),+\infty[.\right.
$$

In fact, consider first the case $p=1$. Let $f: E \rightarrow \mathbb{R}, \bar{x} \in \operatorname{Dom} f$, $y \in A_{\bar{x}}^{f}$ and $\left(x_{n}\right)$ be a sequence converging to $\bar{x}$. There exists a sequence $\left(b_{n}\right)$ such that

$$
\lim _{n \rightarrow+\infty} b_{n}=y \quad \text { and } \quad b_{n} \leq f\left(x_{n}\right), \quad \forall n \in \mathbb{N} .
$$

This yields $y \leq \liminf _{x \rightarrow \bar{x}} f(x)$ and thus

$$
\left.y \in]-\infty, \liminf _{x \rightarrow \bar{x}} f(x)\right],
$$


whence, $\left.\left.\operatorname{cl} A_{\bar{x}}^{f} \subset\right]-\infty, \liminf _{x \rightarrow \bar{x}} f(x)\right]$.

Thanks to Proposition $4.4, A_{\bar{x}}^{f}$ is an interval of $\mathbb{R}$ containing $-\infty$, then to prove the second inclusion, it suffices to show that

$$
\liminf _{n \rightarrow+\infty} f\left(x_{n}\right) \in \operatorname{cl} A_{\bar{x}}^{f} \text {. }
$$

To this end, let $\varepsilon>0$ and $\left(x_{n}\right)$ be a sequence in $E$ converging to $\bar{x}$; there exists $N \in \mathbb{N}$ such that

$$
\liminf _{n \rightarrow+\infty} f\left(x_{n}\right)-\varepsilon \leq f\left(x_{n}\right) \quad \text { for each } n \geq N .
$$

Set

$$
b_{n}:=\left\{\begin{array}{lr}
-\infty & \text { if } n \leq N \\
\liminf _{n \rightarrow+\infty} f\left(x_{n}\right)-\varepsilon & \text { if } n>N .
\end{array}\right.
$$

We have $\lim _{n \rightarrow+\infty} b_{n}=\liminf _{n \rightarrow+\infty} f\left(x_{n}\right)-\varepsilon$ and $b_{n} \leq f\left(x_{n}\right)$ for every $n \in \mathbb{N}$.

This leads to

$$
\liminf _{n \rightarrow+\infty} f\left(x_{n}\right)-\varepsilon \in A_{\bar{x}}^{f}
$$

for all $\varepsilon>0$, accordingly

$$
]-\infty, \liminf _{x \rightarrow \bar{x}} f(x)\right] \subset \operatorname{cl} A_{\bar{x}}^{f} .
$$

Thus,

$$
]-\infty, \liminf _{x \rightarrow \bar{x}} f(x)\right]=\operatorname{cl} A_{\bar{x}}^{f} .
$$

Let us show this inequality for $p>1$. Let $f$ be a mapping defined from $E$ into $\mathbb{R}^{p}$ where $f=\left(f_{1}, f_{2}, \ldots, f_{p}\right)$. We observe that

$$
\begin{aligned}
A_{\bar{x}}^{f} & =\left\{y \in \mathbb{R}^{p}\left|\forall\left(x_{n}\right) \rightarrow \bar{x}, \exists\left(b_{n}\right) \rightarrow y\right| b_{n} \leq f\left(x_{n}\right) \quad \forall n \in \mathbb{N}\right\} \\
& =\left\{\left(y_{1}, \ldots, y_{p}\right) \in \mathbb{R}^{p}\left|\forall\left(x_{n}\right) \rightarrow \bar{x}, \exists\left(b_{n}^{i}\right)_{n \rightarrow+\infty}^{\rightarrow} y_{i}\right| b_{n}^{i} \leq f_{i}\left(x_{n}\right) \quad \forall n \in \mathbb{N}\right\},
\end{aligned}
$$

for $i=1,2, \ldots, p$. This yields

$$
\begin{aligned}
A_{\bar{x}}^{f} & =\left\{\left(y_{1}, \ldots, y_{p}\right) \in \mathbb{R}^{p} \mid y_{i} \in A_{\bar{x}}^{f_{i}} \text { for } i=1,2, \ldots, p\right\} \\
& =\prod_{i=1}^{p} A_{\bar{x}}^{f_{i}} .
\end{aligned}
$$

This product being finite, it follows that

$$
\begin{aligned}
\operatorname{cl} A_{\bar{x}}^{f} & =\operatorname{cl} \prod_{i=1}^{p} A_{\bar{x}}^{f_{i}} \\
& \left.\left.=\prod_{i=1}^{p}\right]-\infty, \liminf _{x \rightarrow \bar{x}} f_{i}(x)\right] .
\end{aligned}
$$


establishing the proof.

Now, we recapture the classical result for finite dimensional valuedfunctions:

Theorem 7.1. The lower limit for finite dimensional valued-functions is lower semicontinuous.

Proof. Take $F=\mathbb{R}^{p}$. It suffices to take $C=\mathbb{R}_{+}^{p}$ and replace $\leq_{c}$ by the usual order $\leq$ in the proof of the main result.

Remark 7.2. Notice here that our proof of this classical result is independent of the closure of the epigraph.

\section{Extension of USUAL OPERATIONS}

To extend the usual operations on the lower and upper limits to the vector case, we first establish the relation between the lower level of the sum of two maps and the sum of their lower level sets.

Proposition 8.1. Let $f$ and $h$ be two vector-valued mappings from $E$ into $F^{\bullet}$ and $\bar{x} \in \operatorname{Dom} f \cap \operatorname{Dom} h$. Then the following inclusions hold:

1) $A_{\bar{x}}^{f}+A_{\bar{x}}^{h} \subset A_{\bar{x}}^{f+h}$;

2) $B_{\bar{x}}^{f}+B_{\bar{x}}^{h} \subset B_{\bar{x}}^{f+h}$.

3) If moreover, $f$ or $h$ is continuous at $\bar{x}$, the inclusions in 1) and 2) become equalities.

Proof. 1) Let $y \in A_{\bar{x}}^{f}+A_{\bar{x}}^{h}$, there exist $y_{1} \in A_{\bar{x}}^{f}$ and $y_{2} \in A_{\bar{x}}^{h}$ such that $y=y_{1}+y_{2}$. Let $\left(x_{n}\right)$ be a sequence converging to $\bar{x}$. As $y_{1} \in A_{\bar{x}}^{f}$ and $y_{2} \in A_{\bar{x}}^{h}$, there exist two sequences $\left(b_{n}^{\prime}\right)$ and $\left(b_{n}^{\prime \prime}\right)$ in $F$ such that

$$
\lim _{n \rightarrow+\infty} b_{n}^{\prime}=y_{1} \quad \text { and } \quad b_{n}^{\prime} \leq f\left(x_{n}\right), \quad \forall n \in \mathbb{N}
$$

and

$$
\lim _{n \rightarrow+\infty} b_{n}^{\prime \prime}=y_{2} \quad \text { and } \quad b_{n}^{\prime \prime} \leq h\left(x_{n}\right), \quad \forall n \in \mathbb{N} .
$$

Set $b_{n}=b_{n}^{\prime}+b_{n}^{\prime \prime}$, we have $\lim _{n \rightarrow+\infty} b_{n}=y_{1}+y_{2}=y$, and

$$
b_{n}=b_{n}^{\prime}+b_{n}^{\prime \prime} \leq f\left(x_{n}\right)+h\left(x_{n}\right), \quad \forall n \in \mathbb{N} .
$$

It follows that $y \in A_{\bar{x}}^{f+h}$.

2) The second inclusion can be established as in 1).

3) Suppose now that $f$ is continuous at $\bar{x}$ and consider a sequence $\left(x_{n}\right)$ converging to $\bar{x}$ and $y \in A_{\bar{x}}^{f+h}$. There exists a sequence $\left(b_{n}\right)$ in $F$ such that

$$
\lim _{n \rightarrow+\infty} b_{n}=y \quad \text { and } \quad b_{n} \leq f\left(x_{n}\right)+h\left(x_{n}\right), \quad \forall n \in \mathbb{N} .
$$


This yields

$$
b_{n}-f\left(x_{n}\right) \leq h\left(x_{n}\right), \quad \forall n \in \mathbb{N}
$$

Let us take

$$
a_{n}=\left\{\begin{array}{cc}
b_{n}-f\left(x_{n}\right) & \text { if } x_{n} \in \operatorname{Dom} f \\
h\left(x_{n}\right) & \text { otherwise }
\end{array}\right.
$$

$f$ being continuous at $\bar{x}$, it follows for $n$ large enough, that $x_{n} \in \operatorname{Dom} f$, and therefore

$$
\lim _{n \rightarrow+\infty} a_{n}=y-f(\bar{x}) .
$$

Hence, we deduce from (8.1) and (8.2) that $y-f(\bar{x}) \in A_{\bar{x}}^{h}$. Thus, $y \in f(\bar{x})+A_{\bar{x}}^{h}$.

As $f$ ist continuous at $\bar{x}, f$ is s-s.c.i at $\bar{x}$. It follows from Proposition 4.1 that $f(\bar{x}) \in A_{\bar{x}}^{f}$, then

$$
y \in A_{\bar{x}}^{f}+A_{\bar{x}}^{h} .
$$

The second inclusion can be concluded analogously.

Theorem 8.2. Let $f$ and $h$ be tow vector-valued mappings from $E$ into $F^{\bullet}$ and $\bar{x} \in \operatorname{Dom} f \cap \operatorname{Dom} h$. The following assertions hold.

1) $v-\lim _{y \rightarrow \bar{x}} \inf f(y)+v-\lim _{y \rightarrow \bar{x}} \inf h(y) \leq_{c} v-\lim _{y \rightarrow \bar{x}} \inf (f+h)(y)$;

2) $v-\lim _{y \rightarrow \bar{x}} \sup (f+h)(y) \leq_{c} v-\lim _{y \rightarrow \bar{x}} \sup f(y)+v-\lim _{y \rightarrow \bar{x}} \sup h(y)$.

3) If moreover $f$ or $h$ is continuous at $\bar{x}$, the inequalities in 1) and 2) become equalities.

Proof. 1) By virtue of Proposition 8.1, we have $A_{\bar{x}}^{f}+A_{\bar{x}}^{h} \subset A_{\bar{x}}^{f+h}$. Let $z \in A_{\bar{x}}^{f}$ fixed; then $z+A_{\bar{x}}^{h} \subset A_{\bar{x}}^{f+h}$, and therefore

$$
\sup \left(z+A_{\bar{x}}^{h}\right) \leq \sup A_{\bar{x}}^{f+h}
$$

Hence,

$$
z+v-\lim _{y \rightarrow \bar{x}} \inf h(y) \leq v-\lim _{y \rightarrow \bar{x}} \inf (f+h)(y),
$$

for all $z \in A_{\bar{x}}^{f}$. Then

$$
\sup _{z \in A_{\bar{x}}^{f}}\left(z+v-\lim _{y \rightarrow \bar{x}} \inf h(y)\right) \leq v-\liminf _{y \rightarrow \bar{x}}(f+h)(y),
$$

which leads to

$$
\sup _{z \in A_{\bar{x}}^{f}} z+v-\lim _{y \rightarrow \bar{x}} \inf h(y) \leq v-\liminf _{y \rightarrow \bar{x}}(f+h)(y) .
$$


Accordingly,

$$
v-\lim _{y \rightarrow \bar{x}} \inf f(y)+v-\lim _{y \rightarrow \bar{x}} \inf h(y) \leq v-\lim _{y \rightarrow \bar{x}} \inf (f+h)(y) .
$$

2) The second inequality can be done similarly.

3) Assume that $f$ is continuous at $\bar{x}$, using Proposition 8.1, we obtain

$$
A_{\bar{x}}^{f}+A_{\bar{x}}^{h}=A_{\bar{x}}^{f+h} .
$$

Thanks to Theorem 4.5, we deduce that

$$
f(\bar{x})-C+A_{\bar{x}}^{h}=A_{\bar{x}}^{f+h} .
$$

Proposition 4.4 implies that

$$
A_{\bar{x}}^{h}-C=A_{\bar{x}}^{h}
$$

which allows to say that

$$
f(\bar{x})+A_{\bar{x}}^{h}=A_{\bar{x}}^{f+h}
$$

whence

$$
\begin{aligned}
\sup \left(A_{\bar{x}}^{f+h}\right) & =\sup \left(f(\bar{x})+A_{\bar{x}}^{h}\right) \\
& =f(\bar{x})+\sup \left(A_{\bar{x}}^{h}\right)
\end{aligned}
$$

Then,

$$
f(x)+v-\lim _{y \rightarrow \bar{x}} \inf h(y)=v-\lim _{y \rightarrow \bar{x}} \inf (f+h)(y) .
$$

Since $f$ is continuous, Theorem 4.11 implies that $A_{\bar{x}}^{f}=f(\bar{x})-C$, and therefore $\sup A_{\bar{x}}^{f}=f(x)$. We conclude that

$$
v-\liminf _{y \rightarrow x} f(y)+v-\liminf _{y \rightarrow x} h(y)=v-\liminf _{y \rightarrow x}(f+h)(y) .
$$

\section{Applichtion to vector-Valued D.C. mappings}

In this section, $H$ is as in Section 6. We shall apply our main result to show that every vector-valued D.C. mapping finite and continuous defined on a Banach space with values in $H$ admits a continuous D.C. decomposition.

We first prove that each finite and continuous vector-valued D.C. mapping admits a lower semicontinuous D.C. decomposition.

Let $\Omega$ be a convex open of $E$. Recalling, for a mapping from $\Omega$ into $H$, the notation $I_{\varphi}(x):=\sup \left(A_{x}^{\varphi}\right)$, we state the following: 
Proposition 9.1. Let $f: \Omega \rightarrow H$ be finite and continuous D.C. vectorvalued mapping on $\Omega$. If $(g, h)$ is a D.C. decomposition of $f$ on $\Omega$, then $\left(I_{g}, I_{h}\right)$ is a D.C. decomposition of $f$ on $\Omega$.

Proof. Let $g$ be an $H_{+}$-convex. At first, we claim that $I_{g}$ is $H_{+}$-convex. In fact, for $\bar{x} \in \operatorname{Dom} g$, as $A_{\bar{x}}^{g}$ is a directed upwards, it follows from Proposition 5.3 that

$$
\left\langle e_{p}, I_{g}(\bar{x})\right\rangle=\left\langle e_{p}, \sup A_{\bar{x}}^{g}\right\rangle=\sup \left\langle e_{p}, A_{\bar{x}}^{g}\right\rangle \quad \forall p \in \mathbb{N} .
$$

According to Lemma 6.2, we have

$$
\sup \left\langle e_{p}, A_{\bar{x}}^{g}\right\rangle=\sup A_{\bar{x}}^{\left\langle e_{p}, g\right\rangle}=\liminf _{x \rightarrow \bar{x}}\left\langle e_{p}, g(x)\right\rangle, \forall p \in \mathbb{N} .
$$

Therefore,

$$
\text { epi }\left(\left\langle e_{p}, I g\right\rangle\right)=\overline{\mathrm{epi}\left\langle e_{p}, g\right\rangle}, \quad \forall p \in \mathbb{N} .
$$

Since $g$ is $H_{+}$-convex, for each $p \in \mathbb{N},\left\langle e_{p}, g\right\rangle$ is convex. This yields $\left\langle e_{p}, I_{g}\right\rangle$ is convex for each $p \in \mathbb{N}$, i.e., $I_{g}$ is $H_{+}$-convex.

Now, let $(g, h)$ be a D.C. decomposition of $f$. Let us show that $\left(I_{g}, I_{h}\right)$ is a D.C. decomposition of $f$ on $\Omega$. For this, let $\bar{x} \in \Omega$, we have

$$
A_{\bar{x}}^{f+h}=A_{\bar{x}}^{g} .
$$

As $f$ is continuous at $\bar{x}$, by Proposition 8.1, we obtain

$$
A_{\bar{x}}^{f}+A_{\bar{x}}^{h}=A_{\bar{x}}^{g} .
$$

On the other hand, Theorem 4.5 leads to

$$
f(\bar{x})-C+A_{\bar{x}}^{h}=A_{\bar{x}}^{g},
$$

and from Proposition 4.4 we have

$$
f(\bar{x})+A_{\bar{x}}^{h}=A_{\bar{x}}^{g} .
$$

Therefore

$$
\begin{aligned}
\sup \left(A_{\bar{x}}^{g}\right) & =\sup \left(f(\bar{x})+A_{\bar{x}}^{h}\right) \\
& =f(\bar{x})+\sup \left(A_{\bar{x}}^{h}\right) .
\end{aligned}
$$

Hence, $f(\bar{x})+I_{h}(\bar{x})=I_{g}(\bar{x})$ for every $\bar{x} \in \Omega$. The proof is complete.

In the scalar case it has been shown [6] that every real D.C. continuous function admits a continuous D.C. decomposition. Here, we provide a generalization for continuous vector-valued maps from a Banach space into a separable Hilbert space. 
Theorem 9.2. Every finite, continuous and $H_{+}-$D.C. mapping on a convex and open subset $\Omega$ of $E$ into a separable Hilbert space ordered by $H_{+}$admits a continuous $H_{+}-D$.C. decomposition on $\Omega$.

Proof. Let $(g, h)$ be an $H_{+}$-D.C. decomposition of $f$. Fix a point $x \in \Omega$. According to Proposition 9.1, $\left(I_{g}, I_{h}\right)$ is a lower semicontinous $H_{+}$-D.C. decomposition on $f$ on $\Omega$. On the other hand, $f$ is continuous at $x$. Then,

$$
x \in \operatorname{Int}(\operatorname{Dom} f)=\operatorname{Int}\left(\operatorname{Dom} I_{g} \cap \operatorname{Dom} I_{h}\right) \subset\left[\operatorname{Dom} I_{g} \cap \operatorname{Int}\left(\operatorname{Dom} I_{h}\right)\right] .
$$

The map $I_{h}$ being $H_{+}$-convex, lower semicontinuous and proper on $\Omega$ and

$$
x \in \Omega \cap \operatorname{Int}(\operatorname{Dom} h),
$$

it follows, for each $p$, that $I_{h_{p}}: H \rightarrow \mathbb{R}$ is convex, lower semicontinuous and proper. Hence, as $\mathbb{R}$ is normal, by Theorem 10.1 below we can conclude that $I_{h_{p}}$ is continuous at $x$, for each $p$ and then so is $I_{h}$. As $g(y)=f(y)+h(y)$ for each $y \in \Omega$, it results that $I_{g}$ is continuous at $x$. The proof is complete.

\section{Appendice.}

Theorem 10.1 (Théra, Ph.D Thesis, 1981). Let $f: E \rightarrow F^{\bullet}$ be a vector mapping. Suppose that $F$ is normal and $f$ is $C$-convex, sl.s.c and proper. If $\operatorname{Int}(\operatorname{Dom} f)$ is nonempty, then $f$ is continuous on $\operatorname{Int}(\operatorname{Dom} f)$.

\section{REFERENCES}

1. Akian, M. (1999), Densities of idempotent measures and large deviations, Trans. Amer. Math. Soc, 351, 4515-4543.

2. Akian, M. and Singer, I. (2003), Topologies on lattice ordered groups, separations from closed downwards and conjugations of type Lau, Optimization, Vol $52,6,629-673$.

3. Borwein, J. M. and Théra, M. (1992), Sandwich theorems for semicontinuous operators, Canad. Math. Bull, Vol. 35, 4, pp. 463-474.

4. Combari, C., Laghdir, M. et Thibault, L. (1994), Sous-différentiel de fonctions convexes composées, Ann. Sci. Math. Québec Vol 18, 2, 119-148.

5. Ellaia, R. and Hiriart-Urruty, J.B. ( 1986), The conjugate of difference of convexe functions, JOTA, Vol 49, 3.

6. Elhilali Alaoui, A. (1996), Caractérisation des fonctions D.C. Ann. Sci. Math. Québec 20, 1, pp 1-13.

7. Fremlin, D.H. (1974), Topological Riesz Spaces and Measure Theory, Lectures in Mathematics, University of Essex. 
8. Gerritse, G. (1997), Lattice-valued semicontinuous functions, Probability and lattices, pp 93-125, CWI Tract 110, Math. Centrum, Centrum Wisk., Inform., Amsterdam.

9. Gierz, G., Hoffmann, K. H., Keimel, K., Lawason, J. D., Mislove, M. and Scott, D. S. (1980), A Compendium of Continuous lattices, Springer-verlag, Berlin, Heidelberg, New York.

10. Holwerda, H. (1989), Closed Hypographs, Semicontinuity and the topological closed-graph Theorem, A unifying Approach, Report 8935, Catholic University of Nijmegen.

11. Lemaire, B. and Volle, M. (1998), Duality in D.C. programming, in Nonconvex Optimization and Its Applications, 27, 331-345. Kluwer Academic Publishers, Dordrecht.

12. Luc, D. T. 1989, Theory of vector optimization, Springer-verlag, Berlin Heidelberg New-York London Paris Tokyo.

13. Martínez-Legaz, J. and Volle, M. (1999), Duality in D.C. programming : the case of several D.C. constraints, J. Math. Anal. Appl, 237, 2, 657-671.

14. Metrane, A. (2003), Applications vectorielles s'écrivant comme différence de fonctions convexes et optimisation sur l'ensemble efficient, Thèse de doctorat, Université Cadi Ayyad, Faculté des Sciences Samlalia, Marrakech.

15. Muselli, E. (2000), Upper and lower semicontinuity for set-valued mappings involving constraints, JOTA, Vol 106, 3, 527-550.

16. Penot, J-P. and Théra, M. (1979), Semi-continuité des applications et des multiapplication, C.R. Acad. Sci. Paris Série A, 419-422.

17. Penot, J-P. et Théra, M. (1982), Semi-continuous mappings in general topology, Archiv der Math, Vol.38, 158-166.

18. Peressini, A. L. (1967), Ordered topological vector spaces, Harper's series in modern mathematics, New-York.

19. Rockafellar, R. T. Wets, R. J-B. (1998), Variationnal Analysis, Springer-Verlag, Berlin Heidelberg New-York.

20. Schaefer, H. H. (1974), Banach lattices and positive operators, Springer-Verlag, Berlin Heidelberg New-York.

21. Théra, M. (1978), Étude des Fonctions Convexes Vectorielles Semi-Continues, Doctorat de Troisième Cycle, N 76, Université de Pau et des Pays de L'Adour.

22. Théra, M. (1981), Subdifferential calculs for convex operators, J. Math. Anal. Appl, vol. 80, 1,78-91.

Mohamed Ait Mansour, Faculté des Sciences et Techniques LACO - UMR 6090 123, avenue Albert Thomas 87060 Limoges cedex.

Abdelmoutalib Metrane, GERAD, Polytechnique Montréal 3000, Chemin de la Côte-Sainte-Catherine Montréal (Québec) Canada, H3T 2A7.

Michel Théra, Faculté des Sciences et Techniques LACO - UMR 6090 123, avenue Albert Thomas 87060 Limoges cedex. 